\title{
Zum historischen Exzerptenwerke des Konstantinos Porphyrogennetos.
}

Auf S. 88-120 d. XV. Bdes der BZ. unternimmt der unserer Wissenschaft unlängst entrissene Theodor Büttner-Wobst den Versuch, die Anlage der historischen Enzyklopädie des Konst. Porphyrogennetos näher zu bestimmen. Ausgehend von den vier Publikationen, die wir als Teile der Enzyklopädie bisher besitzen, nämlich 1. der

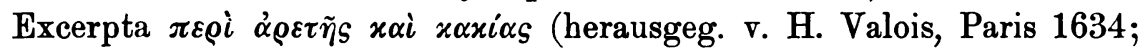

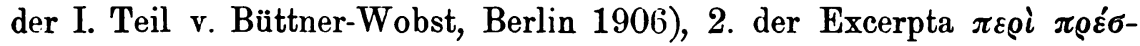
$\beta \varepsilon \omega \nu$ (herausgeg. von de Boor in 2 Bdn., ebda. 1903), 3. der Excerpta $\pi \varepsilon \rho i$ $\nu \omega \mu \tilde{\nu} \nu$ (herausg. v. Boissevain, ebda. 1906) und 4. der Excerpta $\pi \varepsilon \rho \grave{\varepsilon} \pi \iota \beta \rho v \lambda \tilde{\omega} \nu$ (herausg. v. de Boor, ebda. 1906) versucht er einesteils die von Konstantinos exzerpierten Schriftsteller zu einer Liste zusammenzufassen, wobei er bemerkt, daB in seiner Aufzählung nicht alle Autoren genannt sein können, die der Kaiser Konstantin für seine historische Enzyklopädie benutzen lieB, - andernteils ist er bestrebt unter den auf uns gekommenen 24 Titeln, beziehungsweise Sektionen des Konstantinschen Sammelwerkes, eine gewisse Anordnung zu treffen.

Der Natur der Sache gemäB verweilt Büttner-Wobst eines kürzeren auch bei der Exzerptensammlung $\Pi \varepsilon \rho i \delta \eta \mu \eta \gamma o \varrho\llcorner\tilde{\nu} \nu$, die nach seiner Meinung (p. 118) die 28: Sektion der Abteilung über den Staat

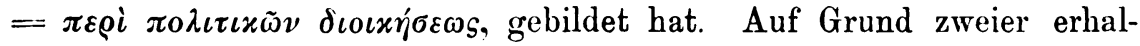

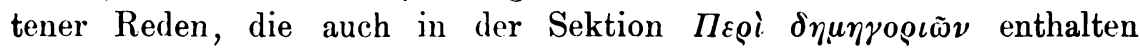

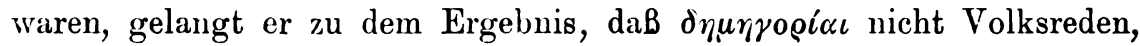
sondern öffentliche Staatsreden gewesen sind. Vielleicht gelingt es uns, mit Anlehnung an die Abhandlung von Büttner-Wobst über diese in Frage stehende Sektion besseren Aufschluß zu erteilen.

Es ist hier nicht der Platz eines näheren auseinanderzusetzen, wie irreführend es heutzutage wäre, wenn jemand auf das von KoechlyRüstow in den Griech. Kriegsschriftstellern II b S. 20 u. ff. Gesagte zurückgreifen würde. Wir sind ja eben durch die Ausgabe der $\Delta \eta \mu \eta$ -

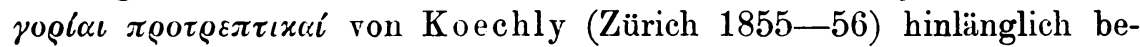
lehrt (s. Krumbacher GBL ${ }^{2}$ S. 260), daB die in mehreren Hss (so Laur. 
LV 4 fol. $218^{\mathrm{r}}-232^{\mathrm{v}}$ und in Abschriften, so Paris. gr. 2522 fol. $78^{\mathrm{r}}$ $110^{\circ}$ und Bern. 97 pag. 153-192 und in der Abschrift des Bernensis Paris. gr. 2446 fol. $68^{\mathrm{r}}-84^{\mathrm{v}}$ ) verstümmelt auf uns gekommene militärische Rhetorik mit dem konstantinischen Exzerptenwerke kaum in $\mathrm{Zu}$ sammenhang gebracht werden kann. Nun will ich versuchen in Folgenden auch den letzten Zweifel in dieser Beziehung aus der Welt zu schaffen.

Die $\Delta \eta \mu \eta \gamma о \varrho i \alpha \iota \pi \rho o \tau \rho \varepsilon \pi \tau \iota x \alpha i$ sind frühestens in Laur. LV 4 enthalten, über dessen kriegswissenschaftliche Sammlung ich in meiner Abhandlung "Zur Überlieferung mittelgriechischer Taktiker" (BZ. XV, S. 47-87) S. 77 u. ff. wahrscheinlich gemacht habe, daB sie im Anfang des X. Jahrhunderts entstanden ist. Schon aus diesem Grunde können sie nicht einen Bestandteil des konstantinischen Sammelwerkes gebildet haben umso mehr, als wir eine ganz andere Masse einer anderen Handschrift kriegswissenschaftlichen Inhalts damit in organischen Zusammenhang bringen können. Aber ich gebe bereitwilligst zu, daB ihr Vorhandensein im ältesten Grundstocke der griechischen taktisch-strategischen Schriften AnlaB gegeben hat, ihr in der zweiten Sammlung Teile des konstantinischen Exzerptenwerkes anzuhängen.

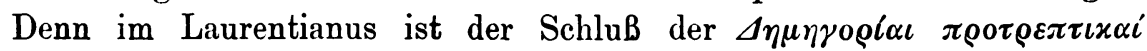
genau so verstümmelt, wie in der Hs., von der sofort die Rede sein soll.

Diese Hs. ist der Ambrosianus B 119 sup., dessen ziemlich genaue Beschreibung zuerst K. K. Müller in der Abhandlung Eine griechische Schrift über Seekrieg (Würzburg 1882) S. 18 u. ff. gegeben hat, und den man neuestens noch genauer in dem prächtigen Catalogus codicum graecorum bibliothecae Ambrosianae von Martini und Bassi (Mediolani 1906) Bd. I. S. 157-160 unter Nr. 139 verzeichnet findet. Schon Müller hat S. 27 die Frage aufgeworfen, ob man nicht in den aus den Historikern exzerpierten Reden auf Folio $141^{\mathrm{r}}-161^{\mathrm{r}}$ Teile des Abschnittes $\Pi \varepsilon \rho i \delta \eta \mu \eta \gamma o \varrho \iota \tilde{\nu} \nu$ der konstantinischen Sammlungen erkennen darf. Ich meine nach der Arbeit von Büttner-Wobst und nach eingehender Betrachtung der weiter unten herausgegebenen konstantinischen $\delta \eta u \eta \gamma o \varrho i \alpha$ ist die von Müller aufgeworfene Frage ganz entschieden mit ja zu beantworten.

In meiner oben zitierten Abhandlung glaube ich den Nachweis geführt zu haben (S. 79), daB die Sammlung, die im Ambros. B 119 sup. vorliegt, auf die Verfügungen des siegreichen Feldherrn Basileios Patrikios zurückgeht. Zugleich habe ich der Meinung Ausdruck gegeben, daB sie um die Mitte des 10. Jahrhunderts entstanden ist, um die Zeit, als der Zug gegen Kreta schon geplant war, eventuell aber auch bald nachher. In der weiter unten veröffentlichten $\delta \eta \mu \eta \gamma o \varrho i \alpha$ 
findet sich nichts, was diese Annahme unwahrscheinlich machen könnte. Der, der das „gottverdammte“ Tarsos so schwer bedrängt hat, war Nıxrøóoos, des Báo $\delta \alpha_{S} \Phi_{\omega x \tilde{\alpha}_{S}}$ Sohn, auf den die kaiserliche Rede hin-

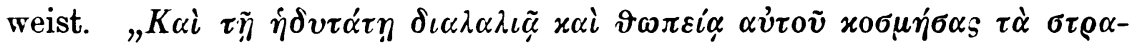

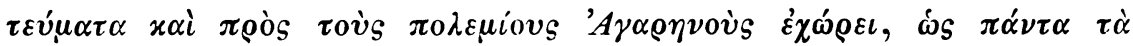

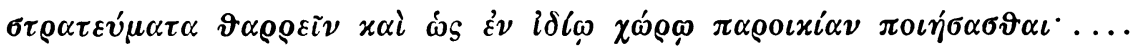

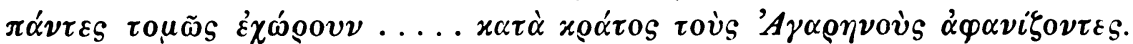

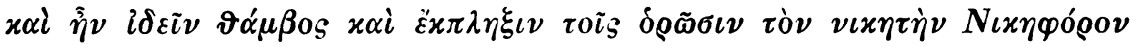

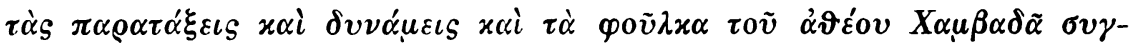

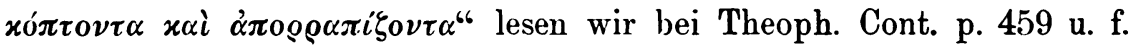
der Bonner Ausgabe. Abu'l Hassan Ali's in unserer $\delta \eta \mu \eta \gamma o \rho l \alpha$ erwähnte zweite Niederlage erwähnt Theoph. Cont. p. 461 ehenfalls: „Tov

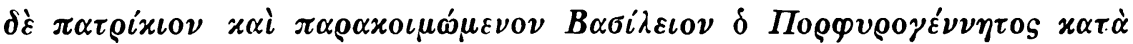

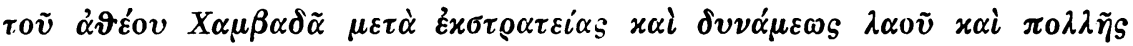

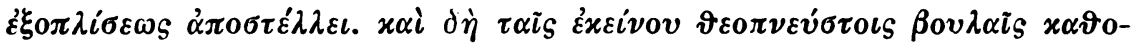

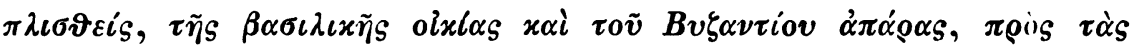

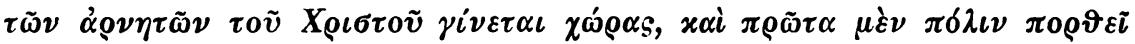

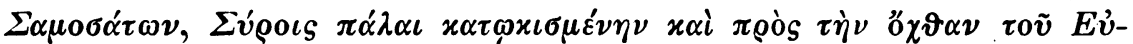

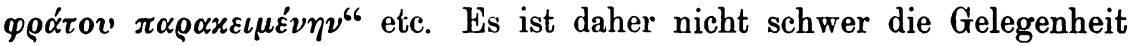
zu bestimmen, wann die Rede gehalten worden ist. Denn bald nachher sendete Konstantin den $\sigma \tau \rho \alpha \tau \eta \gamma \dot{o} \boldsymbol{\tau} \tilde{\omega} \nu \delta v \tau \iota x \tilde{\omega} \nu$ Leon, den Bruder

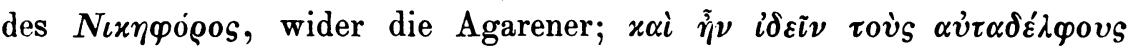

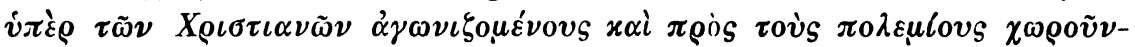

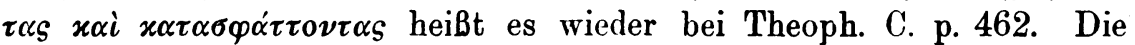
unten veröffentlichte Rede ist daher tatsächlich von Konstantin und nichts beweist, daß sie nicht aus der Exzerptensammlung $\Pi \varepsilon \rho i \delta \eta \mu \eta-$

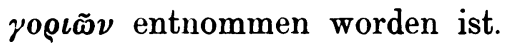

Wir wissen aus der Zusammenstellung der von Konstantin benutzten Autoren, wie sie bei Büttner-Wobst S. 96 zu lesen ist, daB Xenophons Anabasis und Cyropädie sowie Josephos' Bellum Judaicum von Konstantin herangezogen worden sind. Nun bringt unser Ambrosianus aus Xenophons Cyropädie zwei, aus der Anabasis sechs Demegorien, aus Josephos' Belluın Judaicum nicht weniger als vier. Das mag schon an und für sich genügen. $\mathrm{DaB}$ dann nebst einem Anonymus auch noch Herodianus exzerpiert ist, dessen Name sich in der Liste von Büttner-Wobst nicht verzeichnet findet, ist wohl kein Beweis

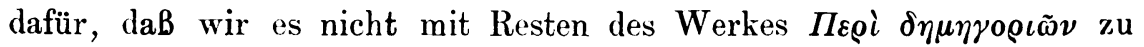
tun haben.

Im Gegenteil, unsere Kenntnis ist jetzt eine vollständigere geworden. Wir wissen nunmehr, daB in dem Werke $\Pi \varepsilon \rho i \delta \eta \mu \eta \gamma o \varrho \iota \tilde{\omega} \nu$ folgende Autoren benutzt worden sind 1. Dio Cassius, 2. Herodianus, 
3. Josephos, 4. Menandros, 5. Nikolaos Damaskenos, 6. Polybios, 7. Theo-

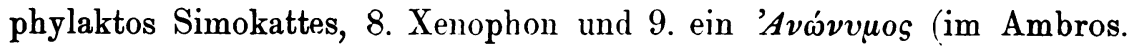
auf Folio $154^{\mathrm{r}}-156^{\mathrm{r}}$ ).

Hierzu kommt dann noch die unten veröffentlichte Rede, die jedenfalls ein ganz prägnantes Beispiel byzantinischer Rhetorik ist. Weitschweifig und voll paränetischer und pathetischer Elemente, mit einem in mancher Hinsicht aus den attischen Tragikern entlehnten Wortschatze, wendet sie sich an Offiziere und Soldaten, denen nur ein geradezu unversiegbarer Wortschwall zu Herzen geht. Versicherung der allumfassenden Liebe des Kaisers zu seinen „Mitstreitern“, Hervorhebung der Tüchtigkeit der Anführer, denen sie unterstellt wurden, Anempfehlung an die Geleitschaft Gottes, Appellation an die Tapferkeit der Streiter, Anspielungen auf die letzthin erfochtenen Siege wider die Ungläubigen, das ist das rhetorische Tiradenwerk, mit welchem der Kaiser operiert, und das seine Demegorie geradezu zu einer militärischen Prunkrede stempelt.

Ich lasse nun hier die Rede folgen so wie sie in der Handschrift überliefert ist; kleinere Versehen des Abschreibers sind dabei stillschweigend verbessert.

[fol. 157 $]$

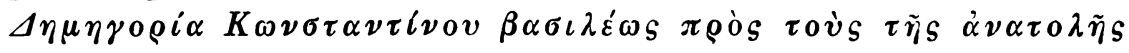
$\sigma \tau \varrho \alpha \tau \eta \gamma \sigma v^{\prime}$.

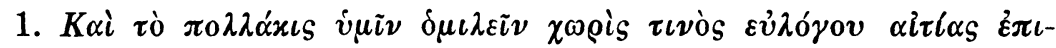

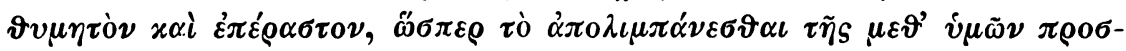

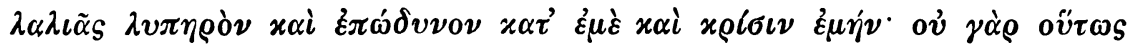

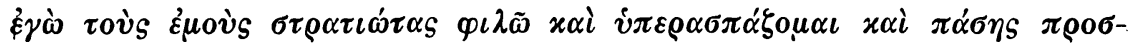

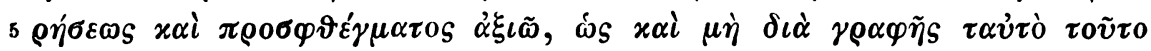

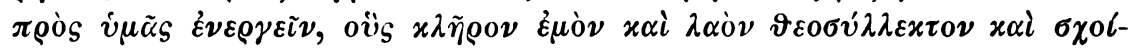

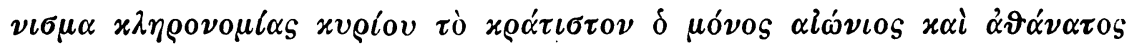

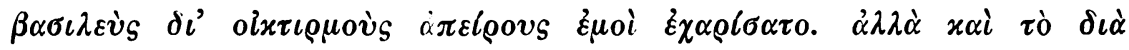

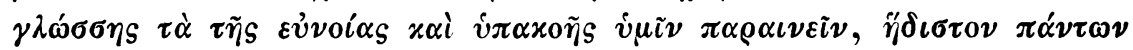

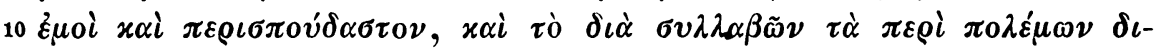

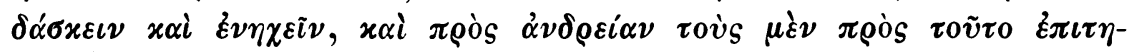

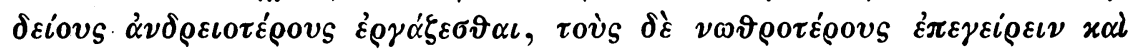

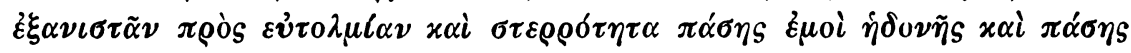

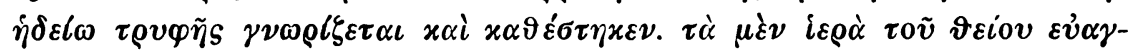

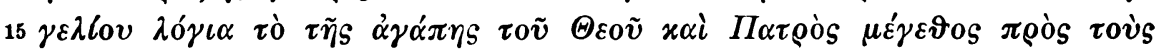

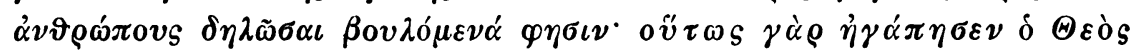

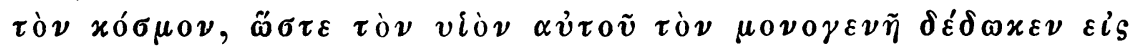

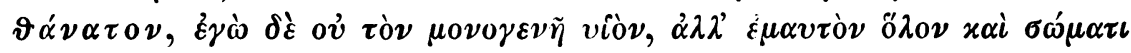




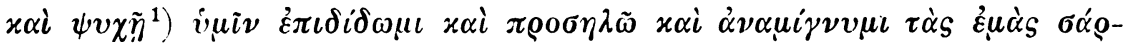

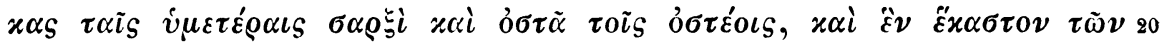

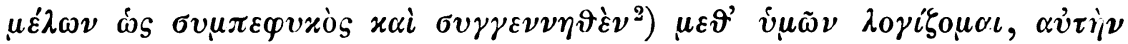

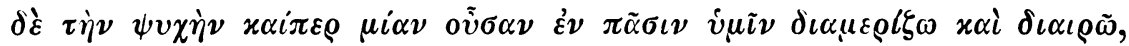

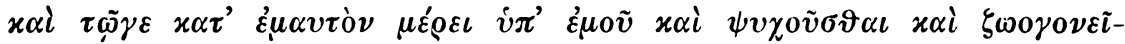

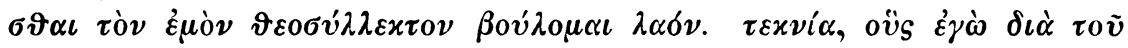

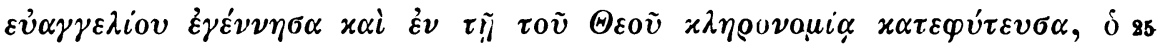

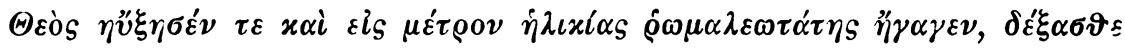

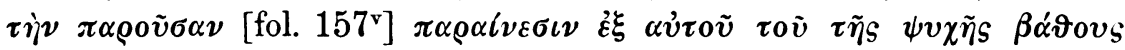

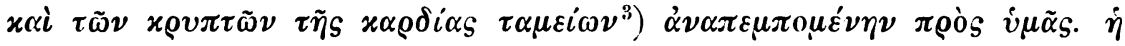

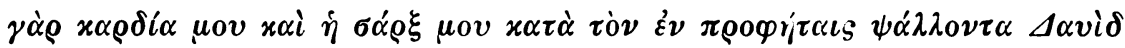

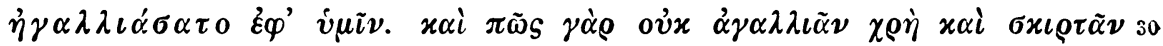

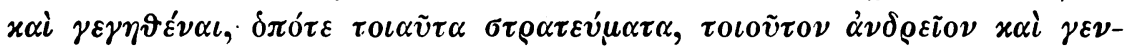

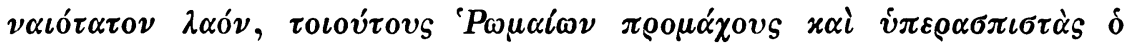

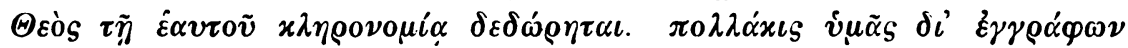

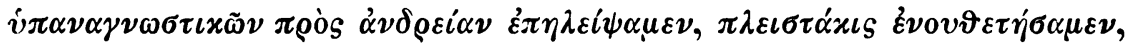
xai xó

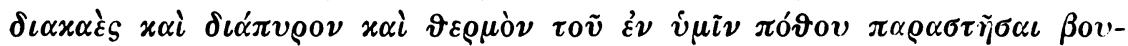

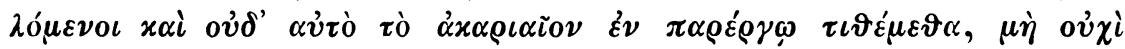

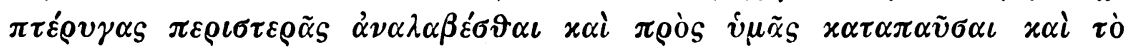

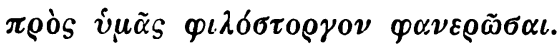

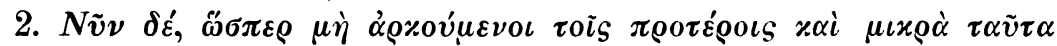

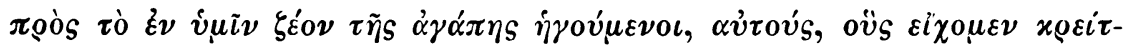

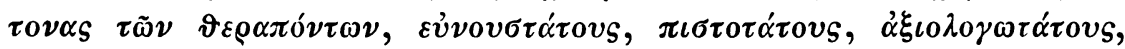

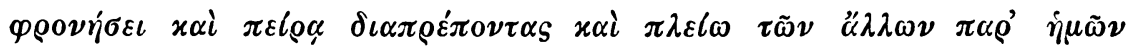

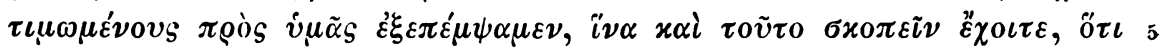

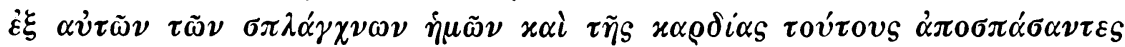

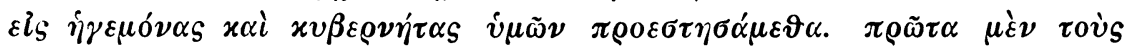

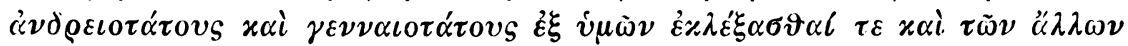

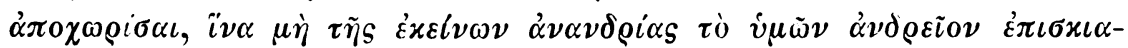

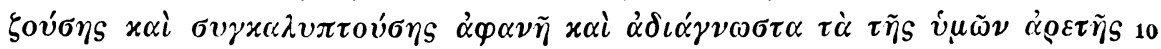

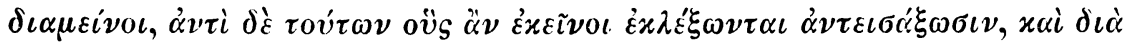

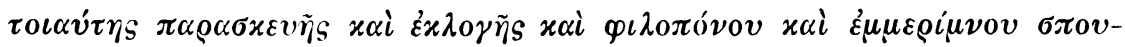

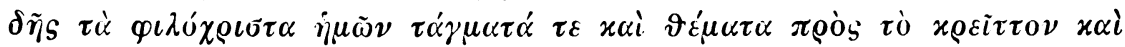

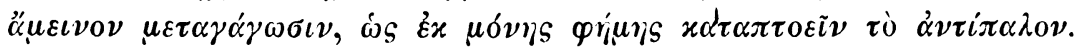

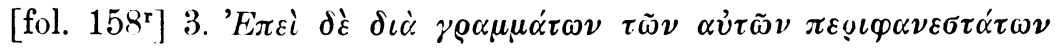

1) Nach $\psi v \chi \tilde{\eta}$ Rasur dreier Buchstaben.

2) $\sigma v \gamma \gamma \varepsilon \nu \eta \vartheta \xi \nu$ die $\mathrm{Hs}$ !

3) Gewiß nicht zu äildern. V'g1. Grönert, Memoria Graeca Herculanensis (Lipsiae 1903) S. 35 u. Ludwich, Anekdota zur griech. Orthogr. IV (Königsberg $1907)$ S. 126. 


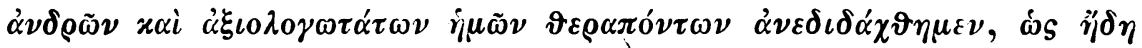

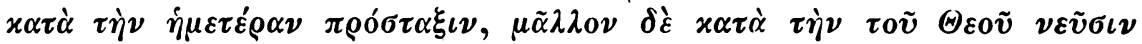

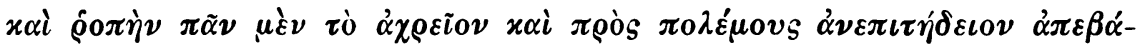

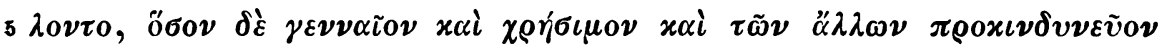

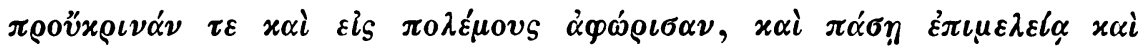

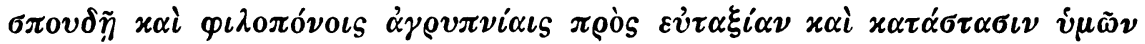

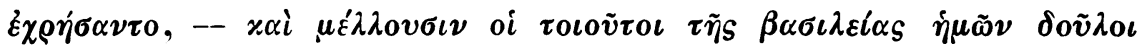

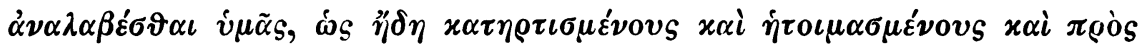

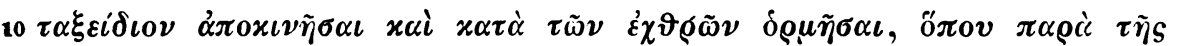

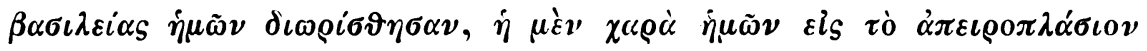

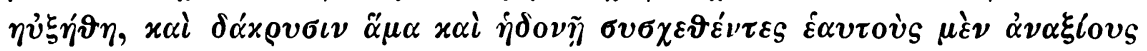

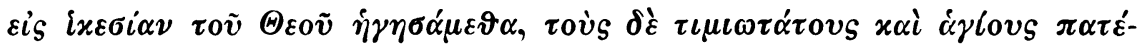

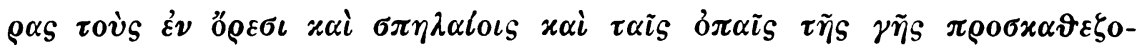

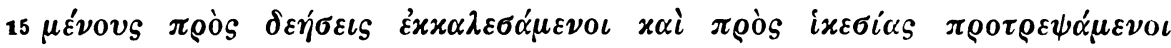

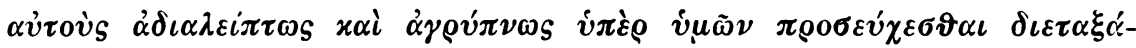

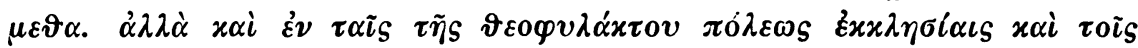

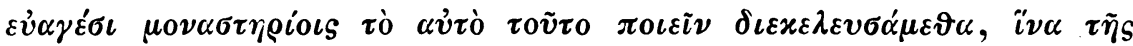

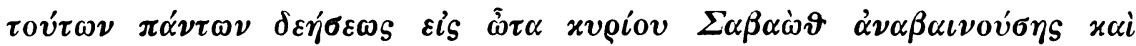

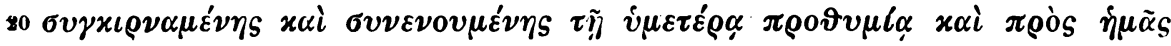

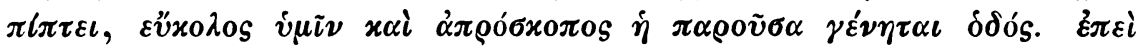

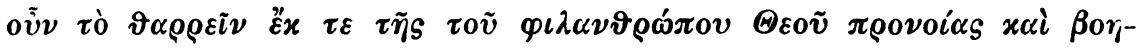

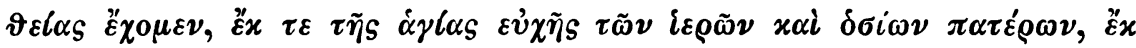

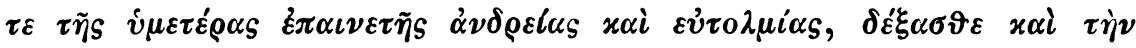

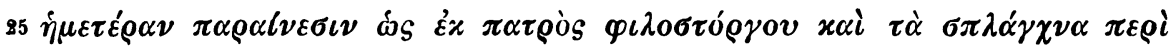

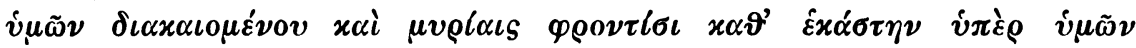

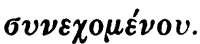

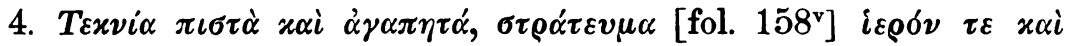

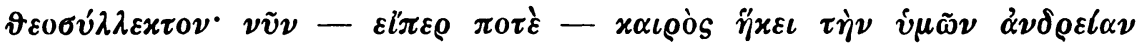

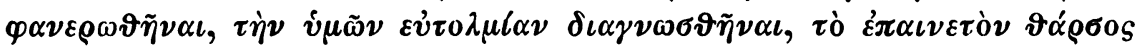

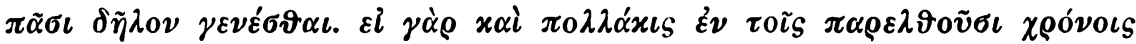

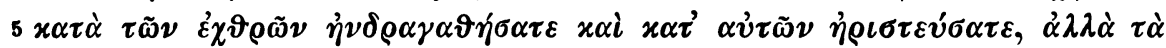

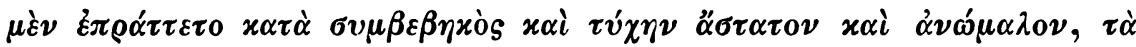

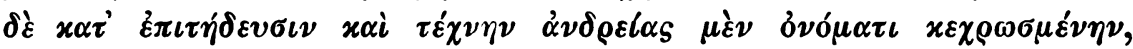

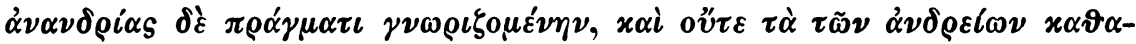

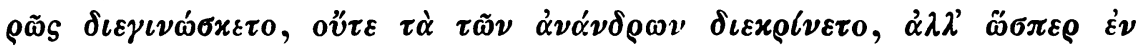

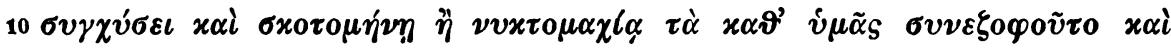

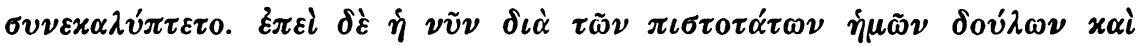

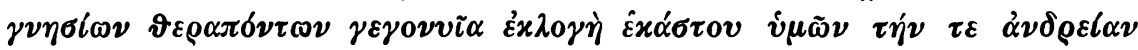

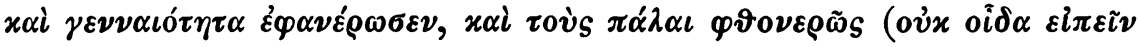

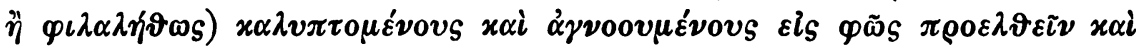

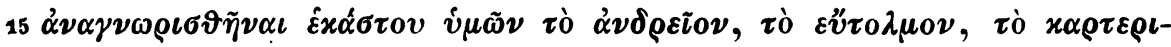




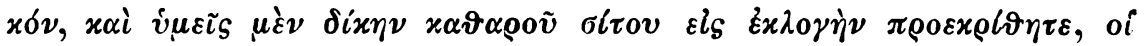

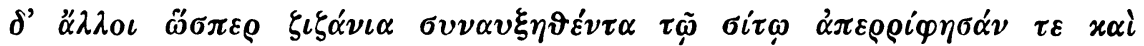

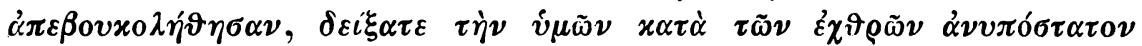

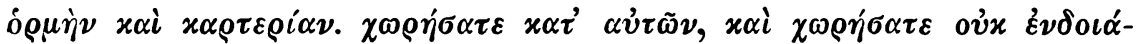

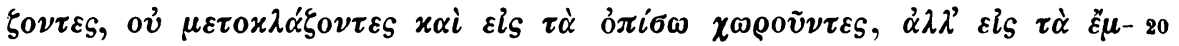

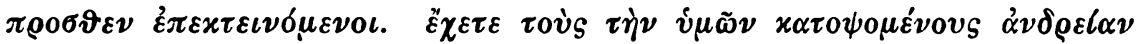

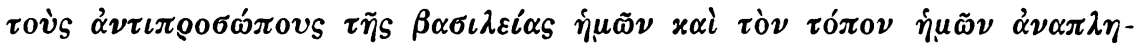

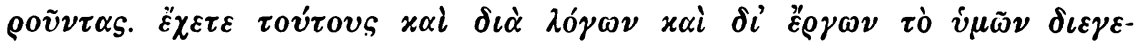

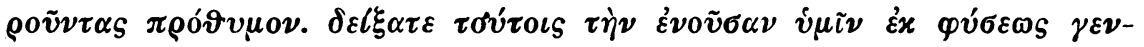

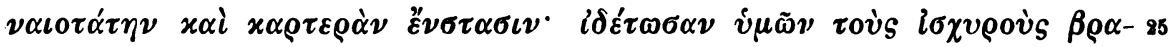

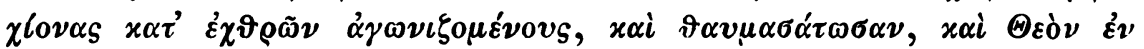

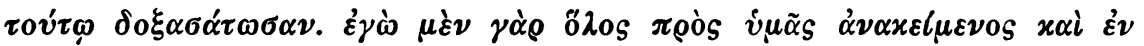

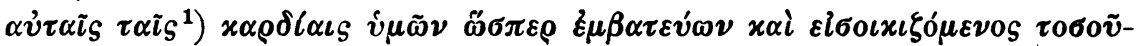

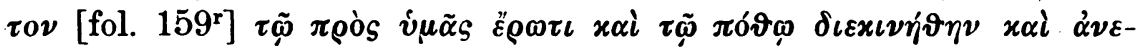

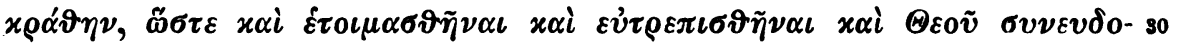

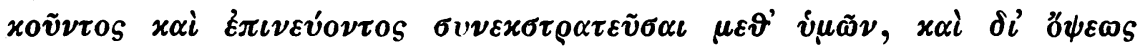

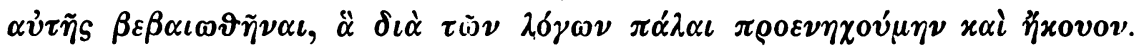

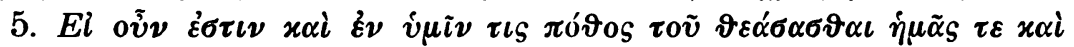

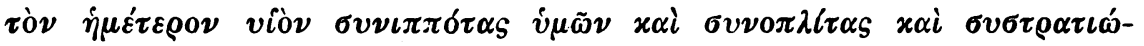

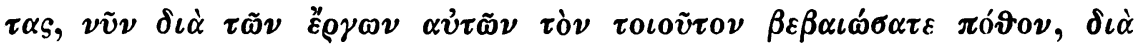

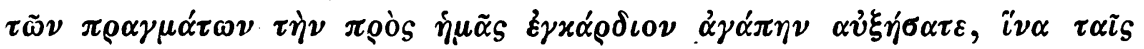

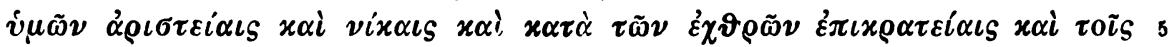

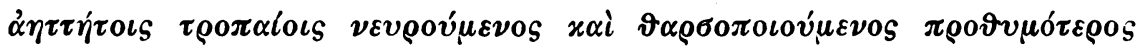

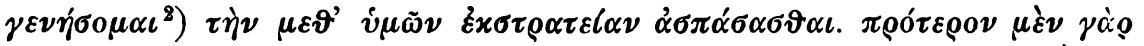

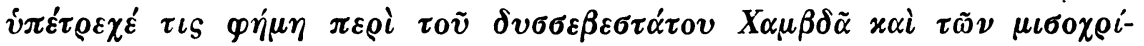

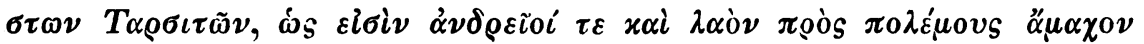

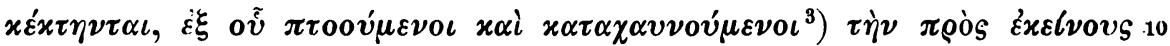

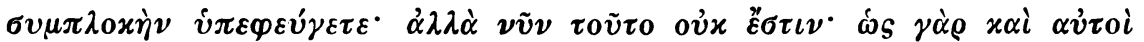

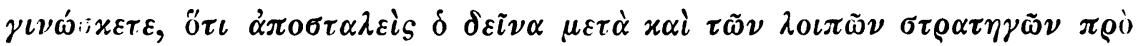

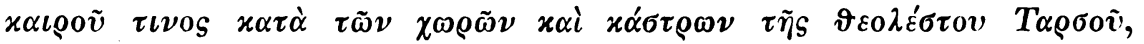

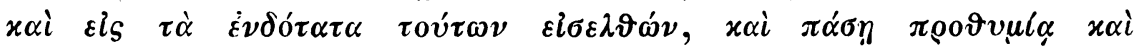

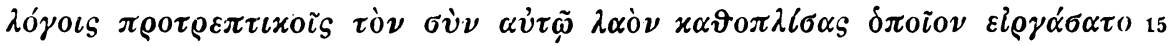

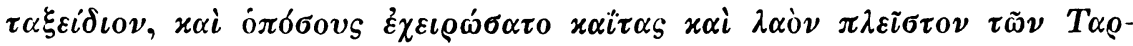

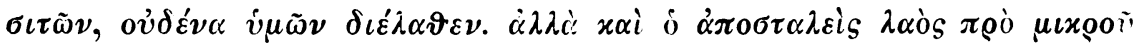

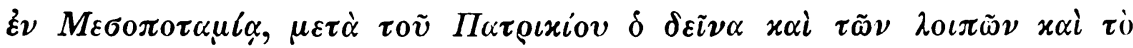

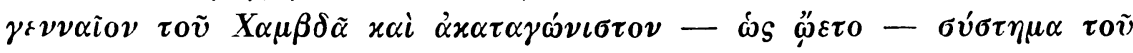

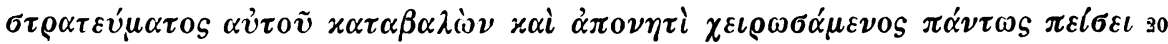

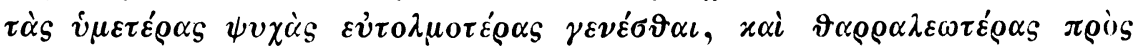

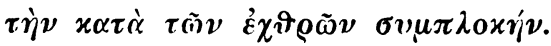
1) $\tau \alpha \sigma \mathrm{A}$. -
2) $\gamma \varepsilon \nu \eta \dot{\sigma} \omega \mu \alpha \iota \mathrm{A}$. -
3) Die Buchstaben oc $x \alpha i$ x $\alpha \tau$ stehen in Rasur. - 


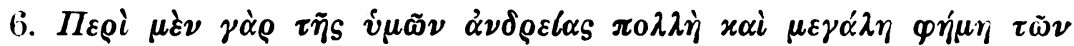

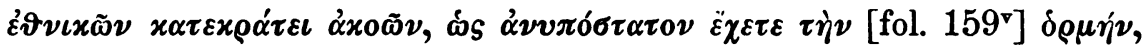

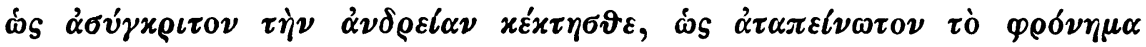

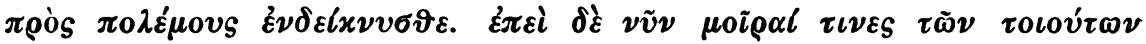

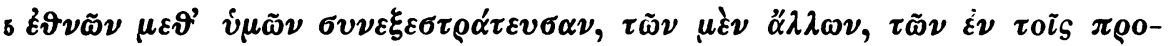

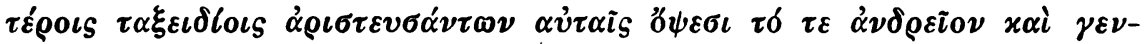

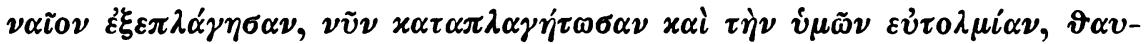

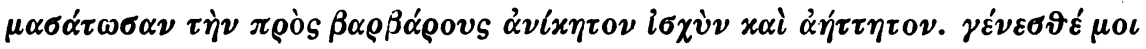

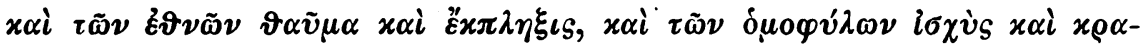

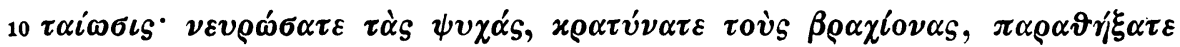

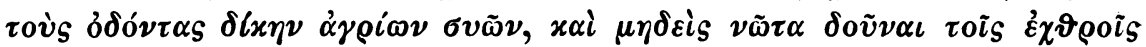

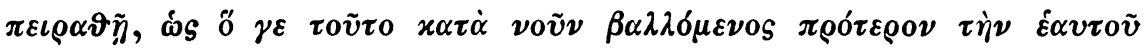

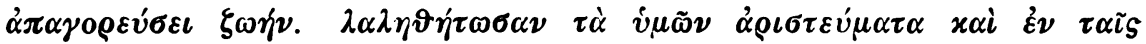

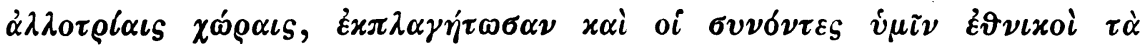

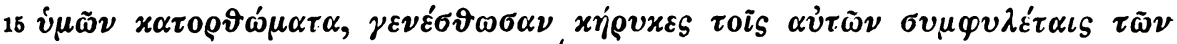

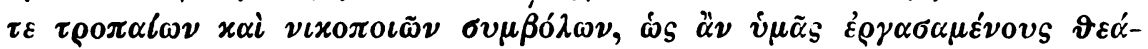
$\sigma \omega \nu \tau \alpha \iota$.

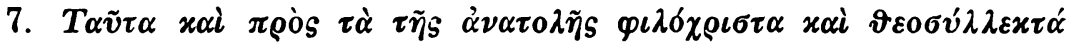

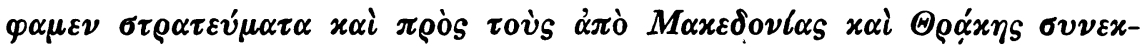

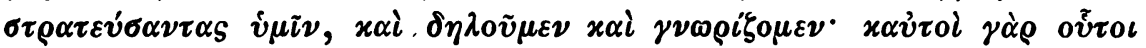

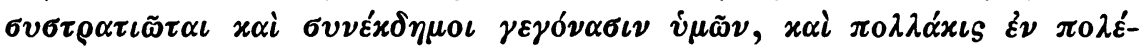

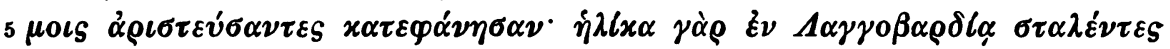

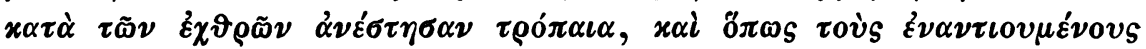

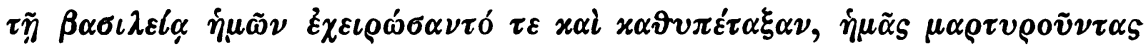
$\pi \varrho 0 \sigma \delta \varepsilon^{\prime} \xi \alpha \sigma \vartheta \varepsilon$.

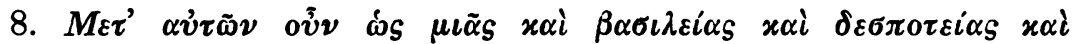

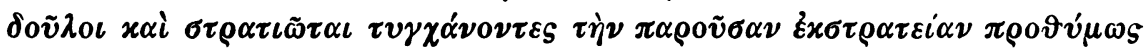

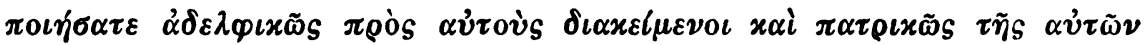

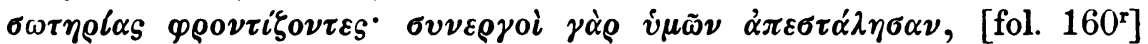

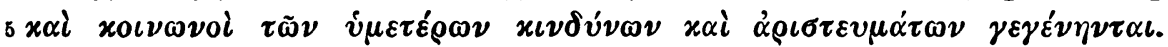

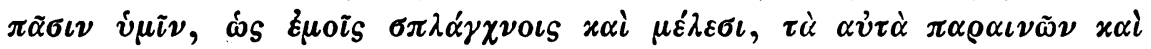

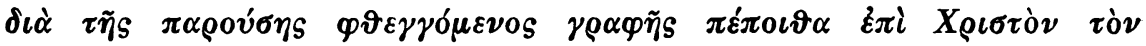

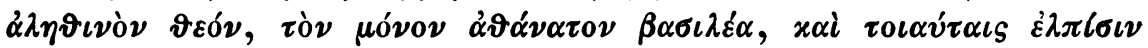

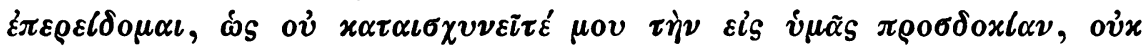

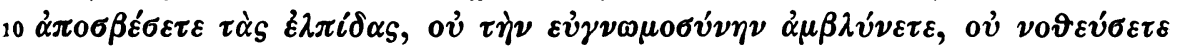

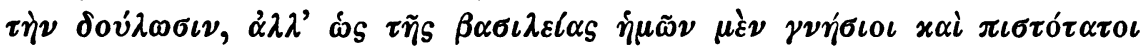

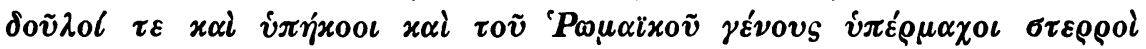

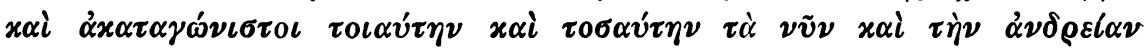

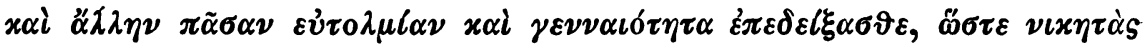

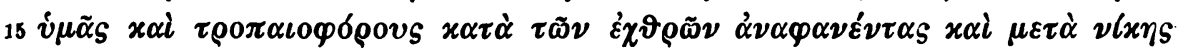

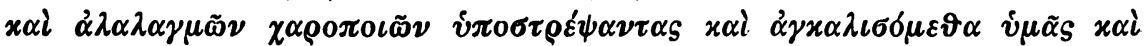




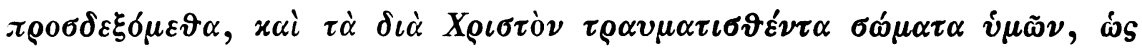

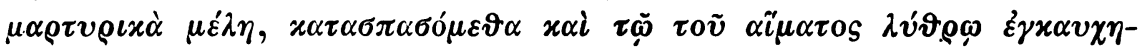

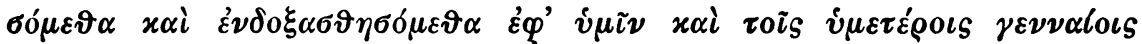

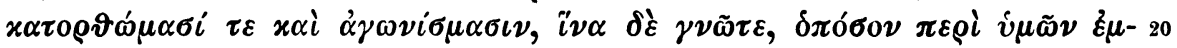

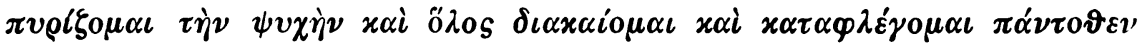

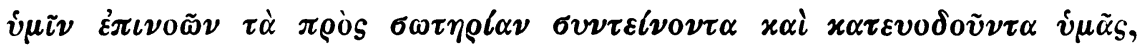

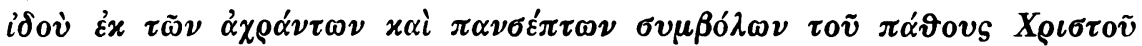

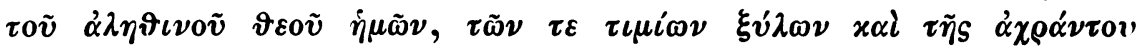

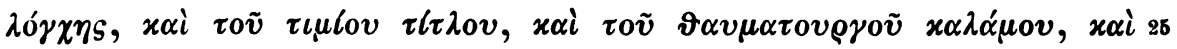

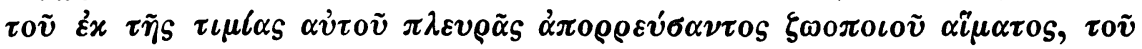

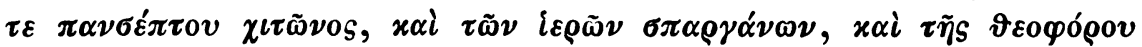

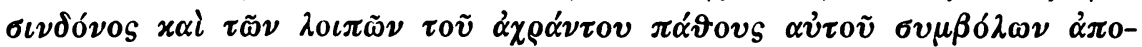

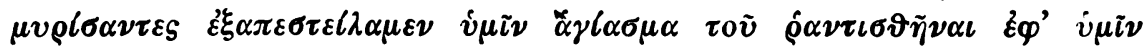

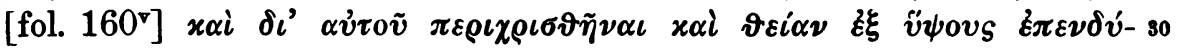

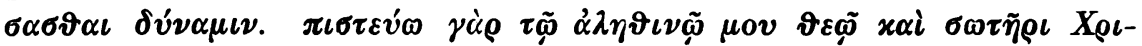

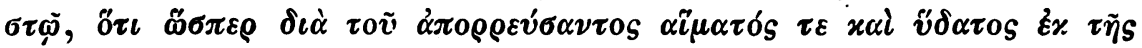

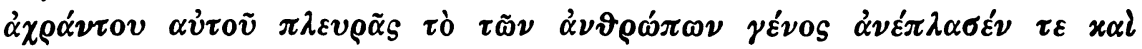

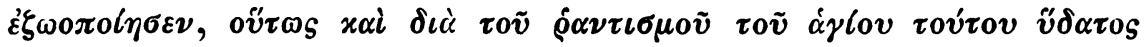

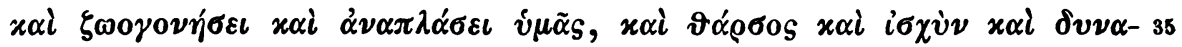

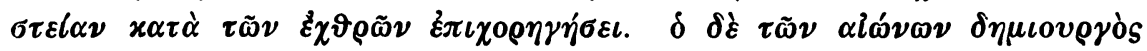

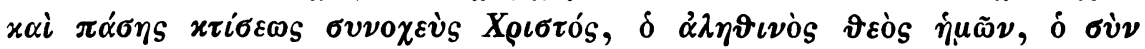

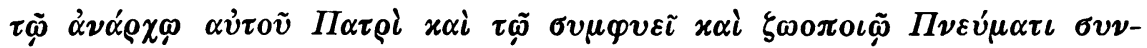

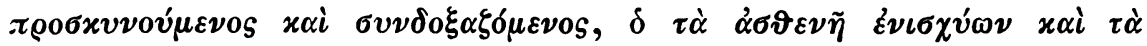

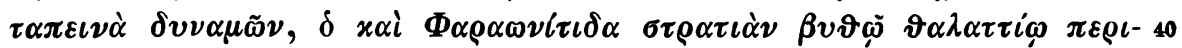

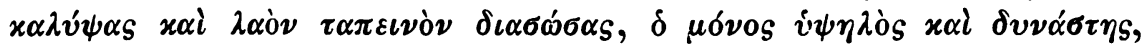

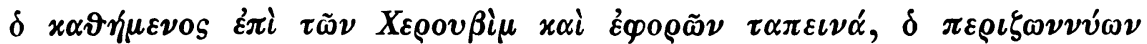

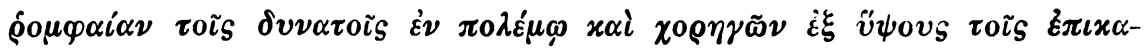

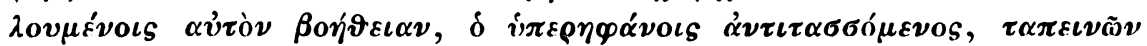

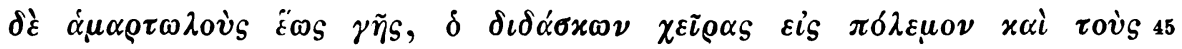

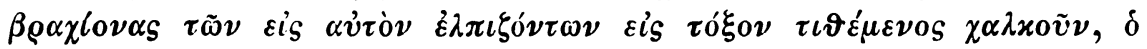

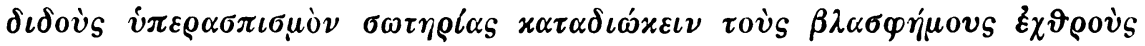

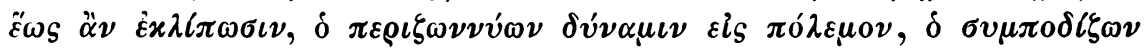

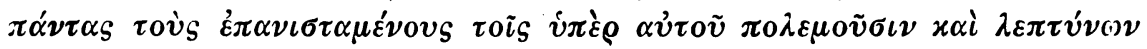

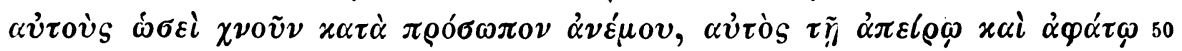

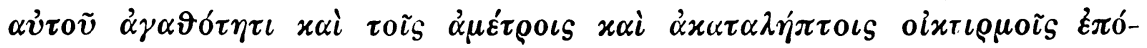

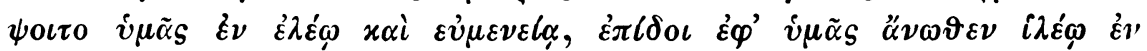

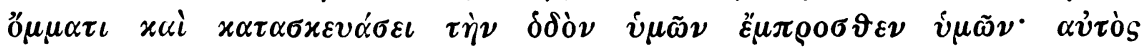

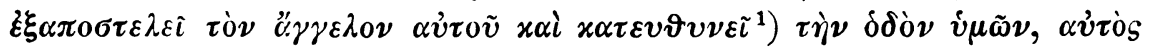

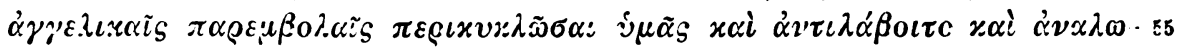

1) $x \alpha \tau \varepsilon v \vartheta v \nu \varepsilon \iota$ die $\mathrm{Hs}$. 


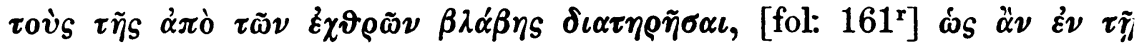

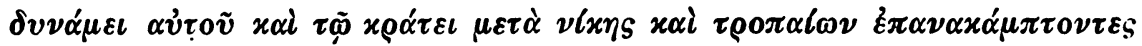

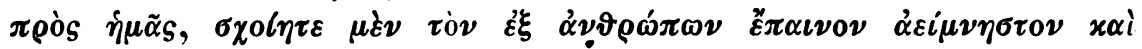

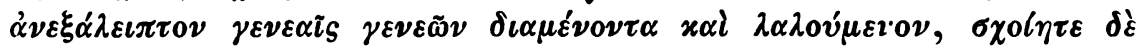

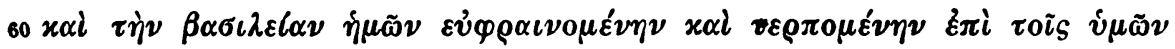

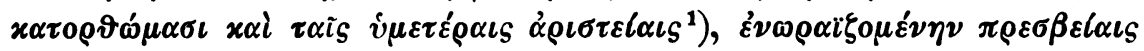

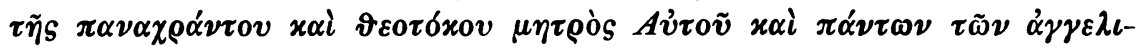

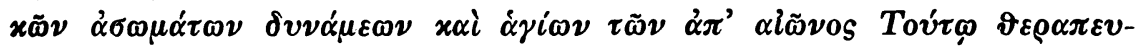

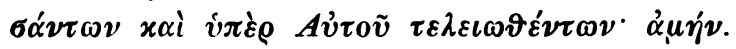

\section{Register.}

$\left.\dot{\alpha} \gamma \alpha \lambda \lambda_{\iota} \alpha^{\prime} \omega\right] \dot{\alpha} \gamma \alpha \lambda \lambda_{\iota} \tilde{\alpha} \nu 1,30, \dot{\eta} \gamma \alpha \lambda \lambda_{\iota} \alpha^{\prime} \sigma \alpha \tau o \quad z=$ in der Bedeutung von $\left.\delta \dot{v} \nu \alpha \mu \alpha \iota\right]$ 1,30

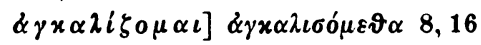

$\dot{\alpha} x \alpha \rho \iota \alpha \tilde{i} 0$ s] $\dot{\alpha} x \alpha \rho \iota \alpha i o v \quad 1,37$

$\left.\dot{\alpha} \lambda \alpha \lambda \alpha \gamma \mu \sigma_{s}\right] \dot{\alpha} \lambda \alpha \lambda \alpha \gamma \mu \tilde{\omega} \nu \quad 8,16$

$\dot{\alpha} \mu \beta \lambda \dot{v} \nu \omega] \dot{\alpha} \mu \beta \lambda \dot{v} \nu \varepsilon \tau \varepsilon 8,10$

$\dot{\alpha} \nu \alpha \tau 0 \lambda \dot{\eta}] \dot{\alpha} \nu \alpha \tau 0 \lambda \tilde{\eta}_{\mathrm{S}} 7,1$

$\dot{\alpha} \nu \varepsilon \xi \dot{\alpha} \lambda \varepsilon\llcorner\pi \tau \circ \mathrm{s}]-0 \nu 8,59$

$\dot{\alpha} \nu \tau \varepsilon\llcorner\sigma \alpha \dot{\alpha} \gamma \omega] \dot{\alpha} \nu \tau \varepsilon \iota \sigma \alpha \dot{\xi} \omega \sigma \iota \nu \quad 2,11$

$\dot{\alpha} \nu \tau i \pi \alpha \lambda_{0}$ s] $\dot{\alpha} \nu \tau i \pi \alpha \lambda o \nu$ 2, 14

$\dot{\alpha} \nu v \pi \delta$ ó $\sigma \tau \alpha \tau o s]-o v 4,18 ; 6,2$

$\dot{\alpha} \pi \varepsilon\left\llcorner\rho \sigma \pi \lambda \alpha^{\prime} \sigma \iota 0 \varsigma\right.$ ] $-0 \nu 3,11$

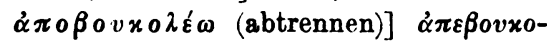

$\lambda \eta^{\prime} \theta \eta \sigma \alpha \nu$ 4, 18

$\dot{\alpha} \pi \circ v \eta \tau i \quad 5,20$

$\dot{\alpha} \tau \alpha \pi \varepsilon i \nu \omega \tau 0 \mathrm{~s}]-0 \nu 6,3$

$\varepsilon \gamma x \alpha v \chi \alpha \dot{\alpha} \beta \mu \iota]-\eta \sigma \delta \mu \varepsilon \vartheta \alpha \quad 8,18$

$\varepsilon \vartheta \nu \iota x o ́ s]$-ol 6,14

$\varepsilon x \lambda \varepsilon i \pi \omega] \varepsilon x \lambda i \pi \omega \sigma \iota v 8,48$

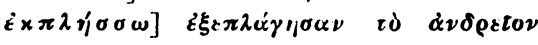

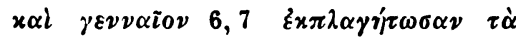

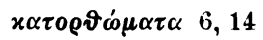

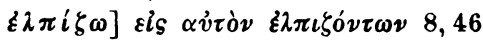

$\xi \mu \beta \alpha \tau \varepsilon \dot{v} \omega] \quad \xi \mu \beta \alpha \tau \varepsilon \dot{v} \omega \nu \quad 4,28$

$\varepsilon \mu \mu \varepsilon \rho \iota \mu \nu 0 s]-i \mu \nu 0 v 2,12$

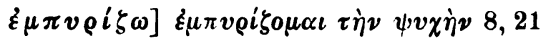

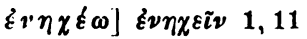

$\xi v \sigma \tau \alpha \sigma \iota s]-\imath \nu 4,25$

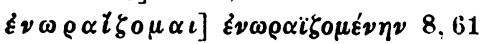

$\left.\xi \pi \alpha \nu \alpha x \alpha^{\prime} \mu \pi \tau \omega\right] \quad \varepsilon \pi \alpha \nu \alpha x \alpha \dot{\alpha} \mu \pi \tau 0 \nu \tau \varepsilon S$ 8, $5 T$

$\varepsilon \dot{\varepsilon} \tilde{\varepsilon} \rho \sigma \tau \tau \sigma \zeta]-0 \nu 1,2$

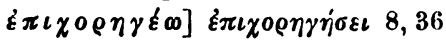

$\varepsilon \dot{v} \gamma \nu \omega \mu \sigma \sigma \dot{v} \nu \eta]-\eta \nu 8,10$

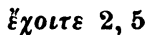

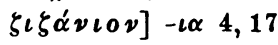

$\zeta \omega \circ \gamma \circ \nu \varepsilon \omega]-\eta \dot{\sigma} \sigma \iota 8,35,-\varepsilon i \sigma \sigma \alpha \iota 1,23$

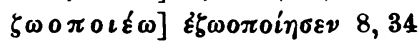

$\zeta \omega 0 \pi \circ i \sigma s]-\tilde{\omega} 8,38$

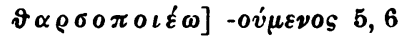

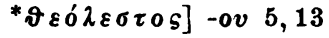

$\vartheta \varepsilon \circ \sigma \dot{v} \lambda \lambda \varepsilon x \tau \sigma s]-o v 1,6 ; 4,2,-\alpha 7,1$

$\Theta \rho \alpha \dot{\alpha} x \eta]-\eta \leq 7,2$

idoi 8,23

$\tilde{i} \nu \alpha$ mit dem Fut. indic. 5, 4

$\left.x \alpha \vartheta v \pi 0 \tau \alpha^{\prime} \sigma \sigma \omega\right] \quad x \alpha \vartheta v \pi \dot{\varepsilon} \tau \alpha \xi \alpha \nu 7,7$

$x \alpha i \tau \eta s] x \alpha i \tau \alpha s \quad 5,16$

$x \alpha \tau \alpha \pi \tau o \varepsilon \dot{c} \omega]-\varepsilon i \bar{\nu} 2,14$

$\left.\left.x \alpha \tau \alpha \sigma \pi \alpha^{\prime}\right\} o \mu \alpha \iota\right] \quad x \alpha \tau \alpha \sigma \pi \alpha \sigma o b \varepsilon \vartheta \hat{\alpha} \quad 8,18$

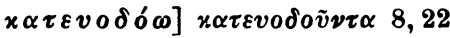

$\Lambda \alpha \gamma \gamma \circ \beta \alpha \rho \delta i \alpha 7,5$

$\lambda \circ \gamma \chi \eta]-\eta s \quad 8,25$

$M \alpha x \in \delta 0 v[\alpha]-l \alpha_{S}$ 7, 2

$M \varepsilon \sigma 0 \pi \circ \tau \alpha \mu i \alpha, 5,18$

$\left.\mu \varepsilon \tau 0 x \lambda \alpha^{\prime} \zeta \omega\right]-\alpha^{\prime} \xi 0 \nu \tau \varepsilon S \quad 4,20$

$\mu \grave{\eta}$ o

$\nu\left\llcorner x \circ \pi 0 \iota \sigma_{s}\right]-\tilde{\omega} \nu 6,16$

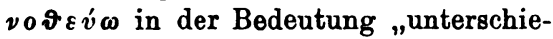

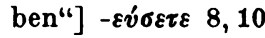

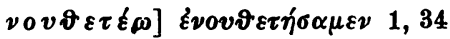

$\delta \delta \varepsilon i \nu \alpha 5,12,18$

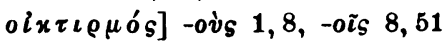

$\delta \pi \eta ́\}] \delta \pi \alpha \tilde{i}_{S} 3,14$

Optativ $x \alpha \tau \alpha \sigma x \varepsilon v \alpha ́ \sigma \varepsilon \iota, 8,53$

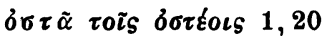

$I ! \alpha \tau \rho \iota x i o v 5,18$

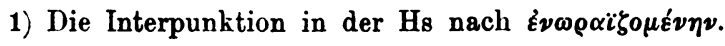




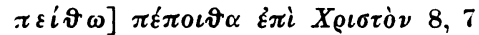

$\pi \varepsilon \rho \iota \sigma \pi \circ v \delta \alpha \sigma \tau o s]-0 \nu 1,10$

$\left.\pi i \pi \tau \omega \pi \sigma_{S} \tau \iota \nu \alpha\right] \pi i \pi \tau \varepsilon \iota 3,21$

$\pi \lambda \varepsilon i \omega$ (vgl. Crönert, Die adverbialem

Komparativformen auf $-\omega$, Philologus

61 [1902] S. $161-192) 2,4$

$\tau \rho \varepsilon \sigma \beta \varepsilon i \alpha$ (Fürbitte)] - $\alpha \iota$ 8, 61

* $\pi \rho \circ \varepsilon \nu \eta \chi \varepsilon \dot{\varepsilon} \omega] \pi \rho \circ \varepsilon \nu \eta \chi 0 \dot{\mu} \eta \nu$ 4, 32

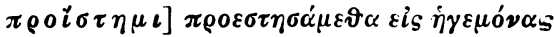

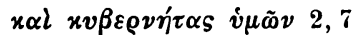

$\pi \rho 0 \sigma \eta \lambda \tilde{\omega} 1,19$

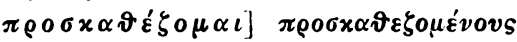

3,14

$\left.\pi \rho \circ \sigma \lambda \alpha \lambda \iota \alpha^{\prime}\right]-\tilde{\alpha}_{S} 1,2$

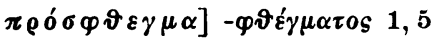

$\oint \alpha \nu \tau i \zeta \omega] \oint \alpha \nu \tau \iota \sigma \vartheta \tilde{\eta} \nu \alpha \iota 8,29$

$\oint \alpha \nu \tau \iota \sigma \mu o ́ s]-0 \tilde{v} 8,34$

$\oint 0 \mu \varphi \alpha i \alpha]-\alpha \nu 8,43$

$\sigma \alpha \beta \alpha \dot{\omega} \vartheta] x v \varrho l o v \sigma \alpha \beta \alpha \dot{\omega} \vartheta \quad 3,19$

$\sigma \iota \nu d \dot{\omega} \nu]$-óvos 8, 28

$\sigma x \iota \rho \tau \dot{\alpha} \omega]-\tilde{\alpha} \nu 1,30$

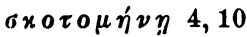

Budapest. $\sigma \pi \alpha \rho \gamma \alpha \nu v \nu]-\omega \nu 8,27$

$\sigma v \nu \varepsilon x \sigma \tau \rho \alpha \tau \varepsilon \dot{v} \omega] \sigma v \nu \varepsilon \xi \varepsilon \sigma \tau \rho \alpha \dot{\tau} \varepsilon v \sigma \alpha \nu 6,5$ $\sigma v \nu \zeta \circ \varphi \delta \omega] \sigma v \nu \varepsilon\} o \varphi o v \tau$ 4, 10

$\tau \alpha \mu \varepsilon \tilde{\imath} 0 \nu]-\varepsilon i \omega \nu 1,28$

$\tau \alpha \xi \varepsilon i \delta \iota 0 \nu] 3,10 ; 5,16,-\varepsilon \iota \delta i ́ o \iota s \quad 6,6$

$T \alpha \rho \sigma i \tau \eta s]-\iota \tau \tilde{\omega} \nu \overline{5}, 9,16$

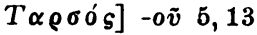

Tempuswechsel 8,10 ov . . $\alpha \dot{\alpha} \mu \lambda v \dot{v} \nu \varepsilon \tau \varepsilon$,

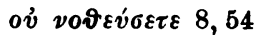

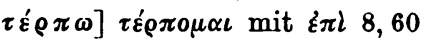

$\tau i \tau \lambda \circ s] \tau i \tau \lambda o v 8,25$

$\dot{v} \pi \alpha \nu \alpha \gamma \nu \omega \sigma \tau \iota x \delta ́ s]-x \dot{\omega} \nu 1,34$

$\dot{v} \pi \varepsilon \rho \alpha \sigma \pi \alpha \zeta \sigma \mu \alpha \iota 1,4$

* $\Phi \alpha \rho \alpha \omega \nu i \tau \iota \varsigma] \quad \Phi \alpha \rho \alpha \omega \nu i \tau \iota \delta \alpha \quad \sigma \tau \rho \alpha \tau i \dot{\alpha} \nu$ 8,40

$\left.X \alpha \mu \beta \delta \alpha^{\prime} \nu\right] X \alpha \mu \beta \delta \tilde{\alpha} 5,8,19$

$\chi \alpha \rho \circ \pi 0 \iota$ ós] $-\tilde{\omega} \nu 8,16$

$\chi \nu \circ \tilde{v}_{S}$ (Flaum)] $\chi \nu \circ \tilde{v} \nu$ 8, 50

$\chi \rho \omega ́(\zeta \omega] \quad \varepsilon \varepsilon \chi \rho \omega \sigma \mu \varepsilon \dot{\nu \eta} \nu 4,7$

ஸs einen Objektsatz einleitend cum ind.

$5,9, \dot{\infty} \gamma \dot{\alpha} \rho 5,11$

R. Vári. 